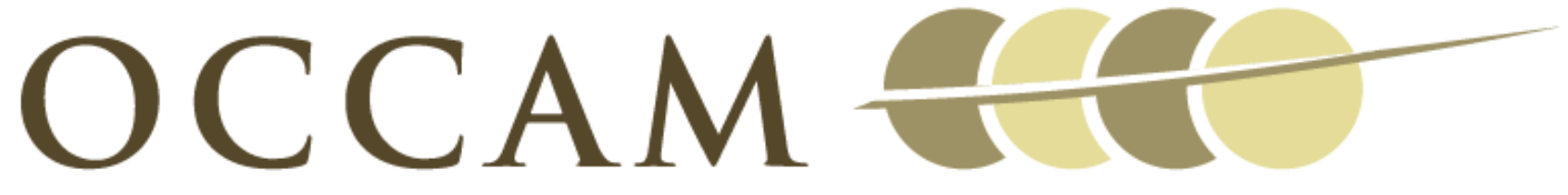

OXFORD CENTRE FOR COLLABORATIVE APPLIED MATHEMATICS

Report Number 10/48

Possible role of differential growth in airway wall remodeling in asthma

by

D.E. Moulton and A. Goriely

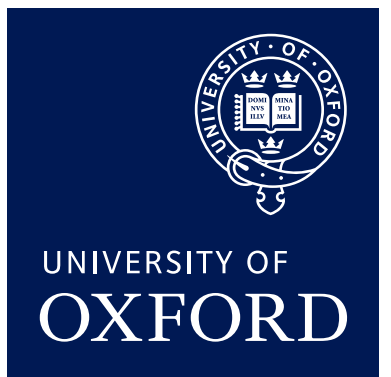

Oxford Centre for Collaborative Applied Mathematics Mathematical Institute 24 - 29 St Giles'

Oxford

OX1 3LB

England 



\title{
Possible role of differential growth in airway wall remodeling in asthma
}

\author{
D.E. Moulton* \\ OCCAM, \\ Institute of Mathematics, \\ University of Oxford, \\ Oxford, UK \\ moulton@maths.ox.ac .uk
}

\author{
A. Goriely \\ OCCAM, \\ Institute of Mathematics, \\ University of Oxford, \\ Oxford, UK \\ goriely@maths.ox.ac.uk
}

November 29, 2010

\begin{abstract}
Airway remodeling in patients with chronic asthma is characterized by a thickening of the airway walls. It has been demonstrated in previous theoretical models that this change in thickness can have an important mechanical effect on the properties of the wall, in particular on the phenomenon of mucosal folding induced by smooth muscle contraction. In this paper, we present a model for mucosal folding of the airway in the context of growth. The airway is modeled as a bi-layered cylindrical tube, with both geometric and material nonlinearities accounted for via the theory of finite elasticity. Growth is incorporated into the model through the theory of morphoelasticity. We explore a range of growth possibilities, allowing for anisotropic growth as well as different growth rates in each layer. Such nonuniform growth, referred to as differential growth, can change the properties of the material beyond geometrical changes through the generation of residual stresses. We demonstrate that differential growth can have a dramatic impact on mucosal folding, in particular on the critical pressure needed to induce folding, the buckling pattern, as well as airway narrowing. We conclude that growth may be an important component in airway remodeling.

keywords: mucosal folding, chronic asthma, growth, elasticity, instability
\end{abstract}

${ }^{*}$ Corresponding author 


\section{Introduction}

Asthma is a disease characterized by a narrowing of the airway and reduced lung function. Chronic asthma is often accompanied by irreversible structural changes to the airway wall, collectively referred to as airway remodeling [43]. Airway remodeling is a complex process occurring at multiple time and length scales and involving many different chemical, biochemical, and physical stimuli. Despite a wealth of research it is still not clear how each of the different structural changes individually affects airway function [51], nor is it known whether these different changes are beneficial or detrimental to asthmatic patients [32]. A well documented key feature of airway remodeling is an increase in airway wall thickness, detected at all levels of the bronchial tree and all layers of the airway wall $[19,22,24,36]$.

In this theoretical paper, we focus on the mechanics of airway wall thickening and the mechanism of mucosal folding at a macroscopic level by building a model based on the most sophisticated constitutive theory for elastic tissue available in the bio-engineering literature. In response to certain stimuli, the smooth muscle surrounding the airway wall contracts, and the luminal boundary folds or buckles - this is the phenomenon of mucosal folding. In "normal patients," such an event is marked by only modest narrowing of the airway [34]. In asthmatic patients, however, this airway narrowing tends to occur for lower stimuli, and also leads to exaggerated narrowing [23].

Mucosal folding is also observed in the esophagus [28, 57], blood vessels [27], and gastrointestinal tract [30]. On a mechanical level, this folding indicates an instability of the inner mucosal edge in response to an external pressure provided by the contraction of smooth muscle. A number of models have looked at mechanical and geometrical aspects of mucosal folding. Some of the key issues from a modeling perspective are: determining the critical buckling pressure, critical buckling mode (i.e. the number of folds in the buckled state), and the degree of airway narrowing; finding relationships between these quantities; and determining the impact of airway wall thickness. Lambert [25] modeled the basement membrane as a single layer elastic tube and showed that the buckling mode can have a dramatic impact on airway narrowing; in particular that an airway with fewer folds will have a greater degree of occlusion if the folds extend until epithelial cells come into contact. This basic model was expanded upon in [26], in which a geometric constraint was included as a mechanism for selecting the number of folds, and was further expanded to include a thin layer of fluid on the inner edge of the airway allowing for possible surface tension effects [15]. Other geometry based models have been proposed, including [47], in which inextensibility of the basement membrane was taken as a geometrical constraint, and the number of folds was directly connected to tethers between the airway and smooth muscle; and [6], which studied effective airway radius given fixed folding geometry. Wiggs et al. put the problem on a more mechanical level in [54], in which the airway was modeled as a bilayered elastic tube. Solving the buckling problem in a finite element analysis, they found wall thickness to have a significant effect on the buckling mode and degree of narrowing. Similar models, also solved with finite elements, were presented a few years later, also incorporating inertial effects of the basement membrane [3] and a comparison with buckling experiments with rubber tubes [18]. 
There are two potential drawbacks to previous models which should be elucidated. First, it is important to note that none of these models allowed for nonlinear material responses to large deformations, which are common in airway narrowing [37]. Over the past decade, it has become increasingly clear in studying the mechanical properties of biological tissues that nonlinear tissue response, inhomogeneity, and remodeling are important, if not crucial, features [8]. The importance of nonlinear elasticity in biological systems is well documented and appreciated in the case of arteries [48, 9, 13, 17, 40], heart [29], muscles [49], brain tissue [55], and many other plants [50, 12] and biological systems [48]. It is clear that in order to understand both the mechanical response and remodeling processes in airways, a constitutive theory based on nonlinear elasticity is required.

Secondly, a potentially important assumption in previous mechanical models is that the mechanical properties of the airway wall do not vary during remodeling, despite evidence that these properties might be altered [21]. That is, airway wall thickness is explored by varying reference dimensions, without any account for how the dimensions might have changed and whether mechanical properties might have varied in the process. This is a key distinction, and underlies the primary question we seek to answer in this paper: could growth significantly impact the mechanical response of the airway to smooth muscle contraction beyond the change in geometry, and thus be an important factor in airway remodeling?

The structural changes involved in airway remodeling fall into the large category of processes known in the bio-engineering literature as growth and remodeling of elastic tissues. Growth in biological systems can be the result of many different processes. Continuum mechanics and the theory of elasticity have long been used to study growth processes and the mechanical properties of growing tissues. It is now understood that biological materials commonly exhibit differential growth, that is the tissue does not grow equally in all directions and/or different parts of the tissue grow at different rates. Differential growth can profoundly alter the geometry and mechanical properties of a material. Local changes of mass induce not only a change in the geometry but also elastic stresses which cannot be eliminated geometrically, and thus generate so-called residual stresses, which persist in the absence of external loads on the material. Residual stress is a hallmark of biological tissues, and plays a key role in the regulation of many biological systems, including arteries [20, 13, 16], blood vessels [7], the human aorta [17], and plant stems [50].

In this paper we model the airway as a two-layer cylindrical structure, and take into account both geometrical and material nonlinearities by utilizing the theory of finite elasticity [39]. The growth of the airway is described via the theory of morphoelasticity, and the buckling of the airway is computed using an incremental deformation stability analysis [2]. As mentioned, the model presented here combines both the nonlinear response of the tissues in large deformation and the effect of growth, features which are absent from previous modeling attempts. (Note Yang et al. recently presented an interesting analysis using the same machinery of finite elasticity for a model of the esophagus [57], but did not consider growth.) Using this model, we demonstrate that differential growth, in particular anisotropic growth, can have a dramatic impact on the critical pressure exerted by smooth muscles needed to trigger buckling, the number of folds, the buckling pattern, and the degree of airway narrowing. Growth 
can also alter stability properties and lead to seemingly counterintuitive results, for example an airway wall may become thicker while losing stability. Our results highlight the importance of mechanical effects due to growth and suggest a need for further experimental research along these lines. Indeed, our hypotheses may be tested by direct measurement of residual stress as noted in [31].

\section{Model and Methods}

We model an airway segment as a bilayer cylindrical structure, considering only tissue interior to the smooth muscle. Following the model given by Wiggs et al. [54], a stiff and thin inner layer corresponds to the mucosal region, consisting of the basement membrane, the lamina propria, and the epithelium [1]. Surrounding this is a portion of the submucosa region, consisting of loose connective tissue. The inner layer is much thinner and stiffer than the outer layer.

The airway smooth muscle (ASM) surrounds the outer layer, with ASM contraction providing a force that deforms and eventually buckles the tube. ASM contraction is a fairly complex process, and several models have been formulated to couple the underlying chemical processes to mechanics $[5,42,53]$. The net mechanical effect of ASM contraction is a normal force applied at the airway wall - smooth muscle interface [42]. Since our purpose here is to focus on growth and buckling, ASM contraction is taken into account as an applied normal pressure boundary condition at the outer edge. Also, since mucosal folding occurs at the mucosal region while the smooth muscle remains roughly circular [26], we impose the boundary condition that the outer edge remain circular in the deformed, buckled state.

The setup is depicted in Figure 1. Material dimensions for the undeformed, reference airway are the inner radius $A$, the thickness of the inner layer, $B-A$, and the thickness of the outer layer $C-B$. We assume an isotropic, incompressible hyperelastic material [4]. To characterize the difference in stiffness between the two layers, the value of the shear modulus is assumed different in each layer, thus we have the parameters $\mu_{1}$ and $\mu_{2}$. From a mechanical perspective, it can be shown that the stiffness ratio $\mu_{1} / \mu_{2}$ is the only important quantity entering the problem.

The model is three dimensional but assumes only plane strain deformation, so that the deformation is uniform along the tube axis. The basic idea behind the analysis is that as the smooth muscle contracts, it creates a pressure normal to the circular cross section and the tube deforms in a symmetric fashion, maintaining its circular shape, until a bifurcation point is reached at a critical pressure, at which point the cylinder "buckles" to an asymmetric state. The buckling analysis follows the incremental theory, as described in [52]. Mathematically, it consists of a linear stability analysis for a solution of mechanical equilibrium in finite elasticity. A nonsymmetric perturbation is added to a symmetric finite deformation, and the equations of mechanical equilibrium are expanded in terms of the perturbation parameter, resulting in a 4th order differential equation for the radial displacement of the nonsymmetric deformation. A solution to the bifurcation equation indicates buckling of the tube. In this formulation, the pressure due to ASM contraction appears as a boundary condition for the initial symmetric deformation while the requirement that the outer edge always remains circular appears 


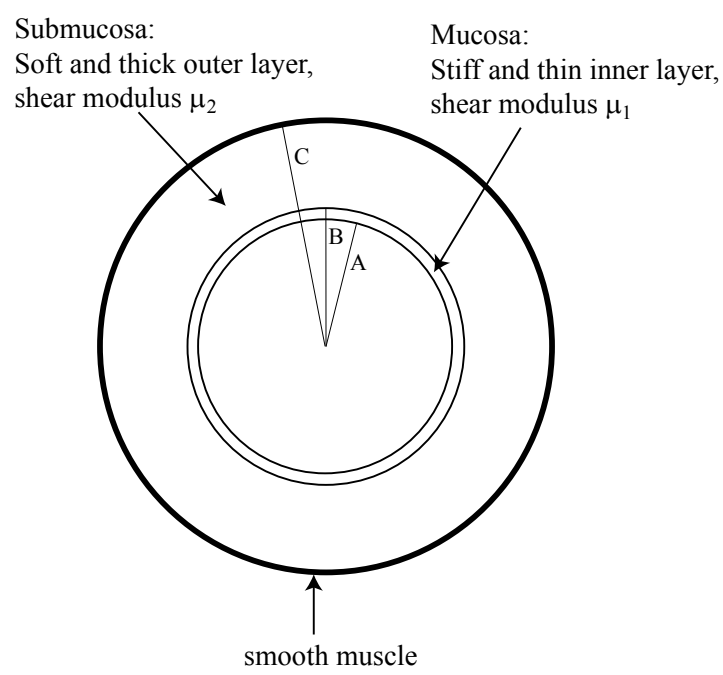

Figure 1: Bilayer model of airway wall, consisting of a stiff and thin inner layer, the mucosa, and a soft and thick outer layer, the submucosa. Surrounding the submucosa is smooth muscle, which applies a normal pressure when it contracts.

as a boundary condition when solving for the nonsymmetric deformation. Also, note the distinction that large deformations and nonlinear tissue response are built into the model by the use of the theory of finite elasticity, but the buckling parameters are determined via a linear stability analysis. This approach has the advantage that buckling properties can be analyzed efficiently without the need for complex and expensive numerical techniques. The drawback, which we return to in Section 4, is that the deformation cannot be tracked beyond the instability. A full derivation of the growth and bifurcation equations, as well as our approach to solving the bifurcation equation, can be found in [35].

The buckling mode number, which is the number of folds in the buckled state, enters as a parameter in the bifurcation equation. For each mode number, a critical pressure is found. This critical pressure represents the pressure necessary to induce buckling at that particular mode. The actual buckling pressure observed in an ideal experiment is the smallest critical pressure over all modes, and the corresponding mode defines the expected buckling pattern.

Growth is included in the analysis via the theory of morphoelasticity and multiplicative decomposition of the deformation tensor $[46,11]$. The basic concept is that the deformation of the body is due to a local change of mass and to an elastic deformation. Since the change of mass is expressed locally, neighboring "cells" can grow differentially. In the absence of elastic deformation, this can induce incompatibilities such as overlapping cells or separation of tissue. The elastic deformation then brings the material back to a compatible configuration; this step can be seen as an elastic 
response to growth, and can induce residual stress in the material.

The effect of symmetric growth in each layer is captured by two parameters per layer, instructing the gain or loss of mass in the radial and circumferential directions. We denote these growth parameters $\gamma_{r}^{(i)}, \gamma_{\theta}^{(i)}, \gamma_{r}^{(o)}, \gamma_{\theta}^{(o)}$. Here $\gamma_{r}$ corresponds to radial growth, with a gain or loss of mass in the radial direction if $\gamma_{r}$ is greater than or less than 1 . The addition or loss of mass in the circumferential direction is captured by $\gamma_{\theta}$, where $\gamma_{\theta}>1$ corresponds to circumferential growth and $\gamma_{\theta}<1$ to circumferential resorption. Figure 2 gives a schematic of radial versus circumferential growth. The superscript differentiates the inner and outer layers. In general, these parameters can be functions of radius, which would signal the case of different "rings" of the airway growing at different rates. Here we consider anisotropic but homogeneous growth in each layer, thus the $\gamma$ 's are constant but not equal.

As our results are largely focused on varying these parameters, it is instructive to further clarify their meaning via a simple example. Consider a single layer tube with radii $A=1, B=2$, with no applied pressure and three different forms of growth:

1. $\gamma_{r}=2, \gamma_{\theta}=2$. The parameter values imply that there is a doubling of mass in both the radial and circumferential directions. Since growth is isotropic and no other forces are applied to the system, the deformed grown tube has radii $a=2$, $b=4$, highlighting a doubling of all radial and circumferential lines. In this case, no residual stress is generated.

2. $\gamma_{r}=1, \gamma_{\theta}=2$. In this case, there is growth only in the circumferential direction. To account for the anisotropic growth, the tube expands to a larger total radius, while keeping nearly the same length of radial lines. A numerical computation gives that the tube after growth has radii $a=2.48, b=3.49$. The growth induces a slight tensile radial stress. There will also be circumferential stress, namely the inner edge will be in tension and the outer edge in compression.

3. $\gamma_{r}=2, \gamma_{\theta}=1$. Here, growth occurs only in the radial direction. A numerical computation gives the radii after growth as $a=0.69, b=2.54$; in this case radial lines want to double in length but are constrained, thus the anisotropic growth induces a compressive radial stress.

To further understand the effect of growth, if we were to plot pressure-area curves (pre-buckling), the area would decrease more rapidly with increased external pressure in Case 2 than in Case 3. This can be understood in terms of the stress induced by the growth. In Case 2, the inner edge is in tension, i.e. there is a positive circumferential stress, whereas the inner edge is in compression in Case 3, corresponding to the compressive radial stress. Thus, in Case 2 the inner edge tends to shrink to relieve the tension, and the area will decrease more rapidly with applied pressure.

\subsection{On material properties}

In this paper we will present results for a neo-Hookean strain energy function $W$, given by $W=\frac{\mu}{2}\left(\alpha^{-2}+\alpha^{2}-2\right)$, where $\mu>0$ is the shear modulus, and $\alpha$ is the circumferential elastic stretch. Lacking data to classify the exact material properties of the 


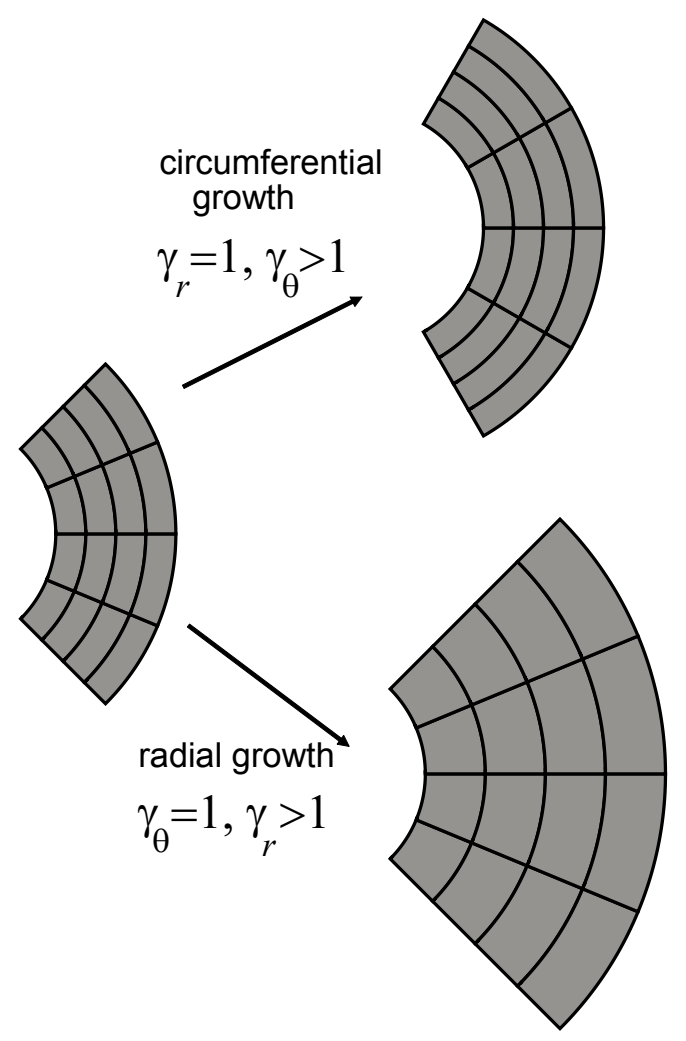

Figure 2: Transformation of an area element under circumferential versus radial growth. 
airway wall, it is worthwhile to check the implications of using a neo-Hookean strain energy. Note that a neo-Hookean material exhibits strain-softening properties. A common strain-energy function for biological tissues which exhibits strain-stiffening is the Fung model, given by $W=\frac{\mu}{2 \beta}\left(\exp \left\{\beta\left(\alpha^{-2}+\alpha^{2}-2\right)\right\}-1\right)$. The parameter $\beta$ typically takes a value between 3 and 20 for soft tissues [10], and the Fung model approaches the neo-Hookean model in the limit of $\beta \rightarrow 0$. In Figure 3, we plot pressure vs area for the symmetric deformation of a bilayered tube for the neo-Hookean model, Fung model with both $\beta=3$ and $\beta=20$, and also for a linear elasticity model. Each model gives qualitatively similar pressure area relationships as expected, but a significant quantitative difference exists between each curve. Thus, the importance of including nonlinearity should be clear; in particular there is a significant difference between the linear model and the Fung model at large pressure. As there is also a significant quantitative difference between the neo-Hookean and Fung curves, the question remains how dependent our results are on the choice of strain-energy. Although the results presented below are only for the neo-Hookean model, we have also explored the same relationships for a Fung model, and have found the same qualitative results, with the quantitative difference that effects are amplified, i.e. a more drastic change in buckling pressure and mode occurs. Since our intent is to provide a general framework for understanding the impact of growth on mucosal folding and asthma, and for a more streamlined presentation, we omit results with the Fung model.

\section{Results}

The bilayer model described above, with 2 growth parameters for each layer, admits a large parameter space. As our goal is to investigate the effect of growth on the buckling of a normal versus a remodeled airway, we will primarily keep reference dimensions of the airway fixed, and change the thickness of the airway by varying the growth parameters. In this way the growth defines a deformation from the normal airway to the remodeled airway. In prior studies, thicker airways have been studied by changing the reference dimensions themselves, without any particular mechanism to account for the change. By keeping the reference dimensions fixed and altering the growth parameters, we can explore the effect of thickness on the buckling, but we are able to account for the change of thickness as well as the changes in material properties resulting from additional residual stresses. We use as reference "normal airway" dimensions the base values $A=0.98, B=1, C=1.5$, and stiffness ratio $\mu_{1} / \mu_{2}=10$. These values are motivated by physiological measurements [22] and have been used in previous airway models [54, 18]. It should be noted that obtaining precise values for thickness and stiffness ratios is a challenging task and varies over generations of the bronchial tree. Thus, we also explore the effect of changing reference dimensions in Section 3.5.

\subsection{Isotropic growth}

The first effect we consider is isotropic growth, but with different growth rates in each layer. That is, we assume $\gamma_{r}=\gamma_{\theta}$ in each region but that $\gamma^{(i)} \neq \gamma^{(o)}$. As an example, we let $\gamma^{(o)}=1.2$ and vary $\gamma^{(i)}>1$. We plot in Figure 4 the critical pressure (a) and critical 


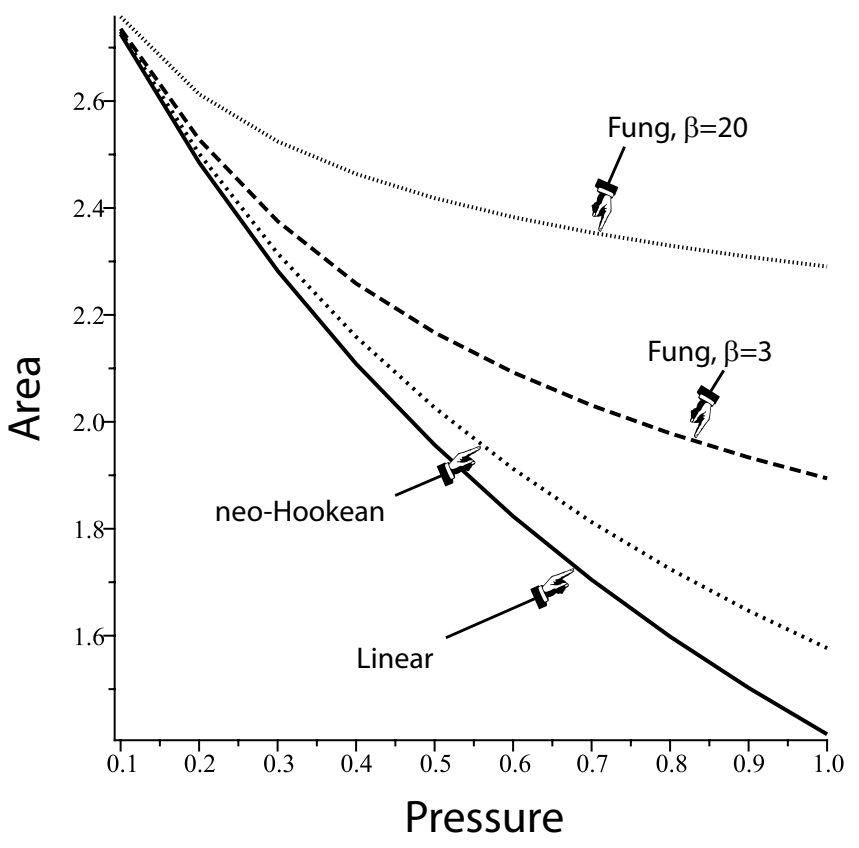

Figure 3: A comparison of pressure vs area for the symmetric deformation of a bilayered tube for 4 different forms of model. Parameters are $A=0.98, B=1, C=1.5$, and $\mu_{1} / \mu_{2}=10$. 
buckling mode (b) as a function of $\gamma^{(i)}$ ranging from 0.9 to 1.4. In Figure 5 the same plots are shown with $\gamma^{(i)}=1.2$ fixed and $\gamma^{(o)}$ ranging from 0.95 to 1.5 . It should be noted that the critical buckling pressure only depends on the ratio $\gamma^{(i)} / \gamma^{(o)}$. Different values with the same ratio are equivalent up to an isotropic and equal growth multiple in each layer, so that the critical pressure does not differ (note that the airway dimensions will vary by the same scalar multiple). Thus, the critical pressure for $\gamma^{(i)}=1.2$ in Figure 4, and for $\gamma^{(o)}=1.2$ in Figure 5, corresponds exactly to the critical pressure in the absence of growth (as shown by the horizontal line on the figures). Comparing the critical pressure to this reference case, it is interesting to note that when the ratio $\gamma^{(i)} / \gamma^{(o)}>1$, the airway is less stable, i.e. it buckles at a lower pressure. On the other hand, a greater pressure is required when this ratio is less than one. In other words, the airway is weaker with respect to buckling when the inner layer grows at greater rate than the outer layer, and likewise stronger when the outer layer grows faster. Observe that this effect is significant as the change in critical pressure increases by a factor of about 8 as $\gamma^{(o)}$ changes from 1 to 1.5 .

As stated, the linear stability analysis enables us to find critical buckling parameters, but once buckling occurs we have no direct information on the magnitude of the deformation. Thus, we cannot comment directly on the exact amount of narrowing of the airway after buckling occurs. Nevertheless, we can make relative comparisons of the size of the non-symmetric deformation for different parameters. In this way, we can determine whether buckling at one set of parameters should result in more or less narrowing (initially) than buckling at another set of parameter values. Buckling patterns for the points marked I-IV in Figures 4(a) and 5(a) are shown in Figure 6. Comparing I and II, in the case of $\gamma^{(o)}=1.2$ fixed, each type of growth can be argued to have advantageous and disadvantageous features. At the lower value of $\gamma^{(i)}$, the buckling pressure is much higher but the airway narrows significantly more. If an airway were to grow with the growth rates at point I, it would have a greater resistance to ASM contraction but would have exaggerated narrowing when buckling occurs. At the higher value, it is the opposite: the airway narrowing is reduced but the buckling pressure is much lower. There is essentially a trade off between the two growth rates. This trade off does not occur in the case of fixed $\gamma^{(i)}$ (Figure 5). The larger value of $\gamma^{(o)}$ (point IV) has a higher resistance to buckling and comparable narrowing to the smaller value of $\gamma^{(o)}$ (point III). Note that the growth ratio is nearly equal at points I and IV, but both values of $\gamma$ are higher at IV, and the increased growth leads to a larger airway area. Between the destabilizing growths, III is more detrimental to airway function as it has greater airway narrowing.

Referring to Figures 4 - 5, it seems counterintuitive that more growth in the outer layer is required to strengthen the airway wall, since the inner layer is stiffer. In fact, a primary result of Wiggs et al. [54] was that increasing the thickness in the inner layer has a greater impact on buckling than increasing the thickness of the outer layer. The reasoning behind this is that differential growth creates residual stress, which induces a competition between mechanics and geometry. In particular, when the inner layer grows, it pushes against the outer layer, creating a compressive residual stress in each layer. This mechanical effect is destabilizing against external pressure, so that even though geometrically the tube may seem stronger, this is outweighed by the mechanical destabilization. (Conversely, when the outer layer grows faster, it pulls the 
(a)
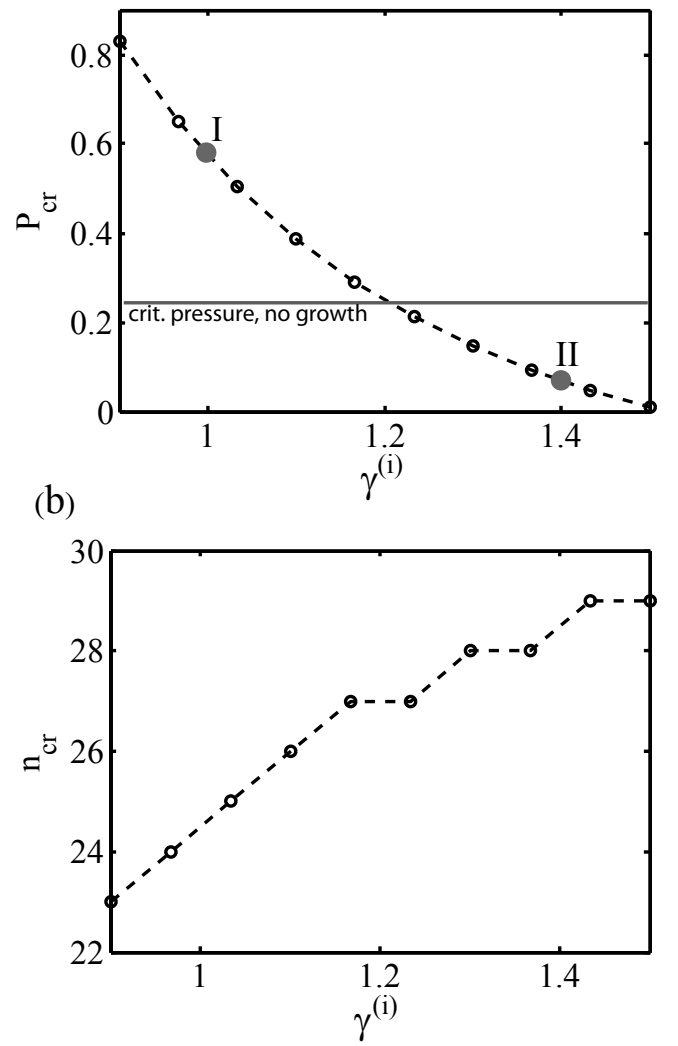

Figure 4: Critical buckling pressure (a) and buckling mode (b) as a function of isotropic growth of the inner layer, for fixed isotropic growth in the outer layer, $\gamma^{(o)}=1.2$. 

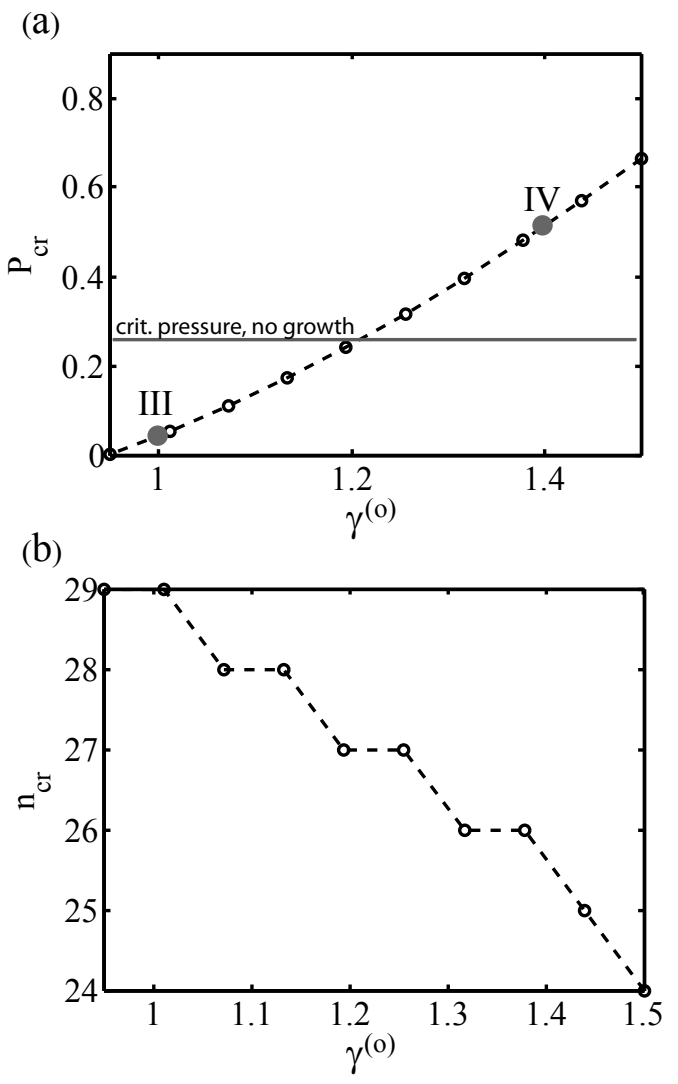

Figure 5: Critical buckling pressure (a) and buckling mode (b) as a function of isotropic growth of the outer layer, for fixed isotropic growth in the inner layer, $\gamma^{(i)}=1.2$. 

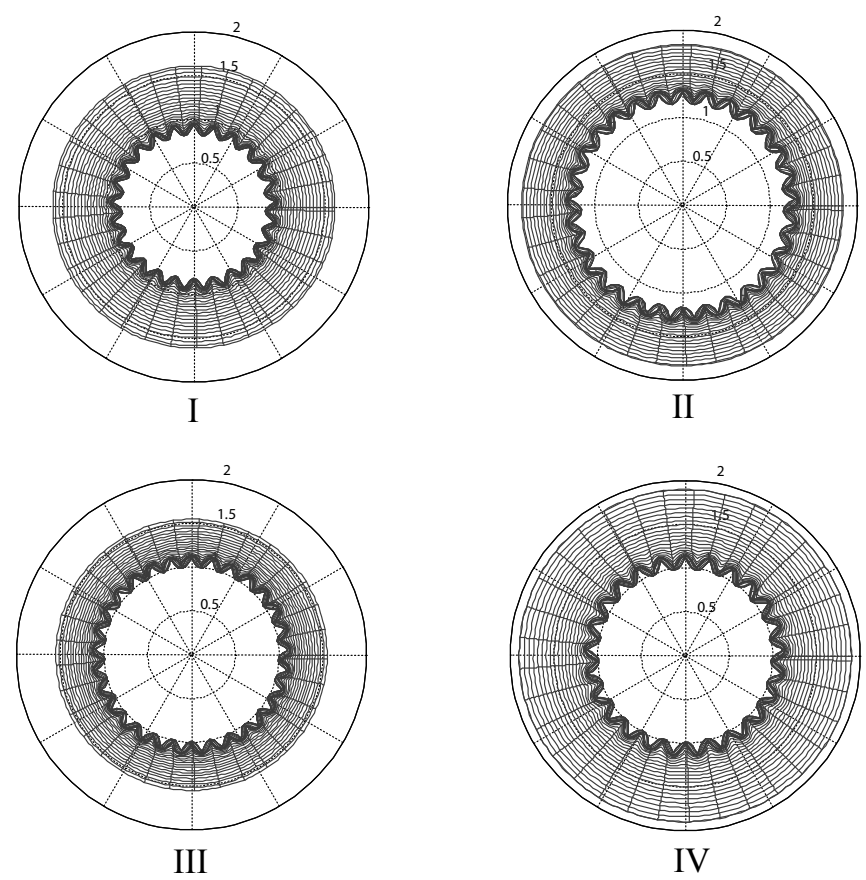

Figure 6: Buckling patterns, or tube deformation, after bifurcation for the points marked I-IV in Figures 4(a) and 5(a). 


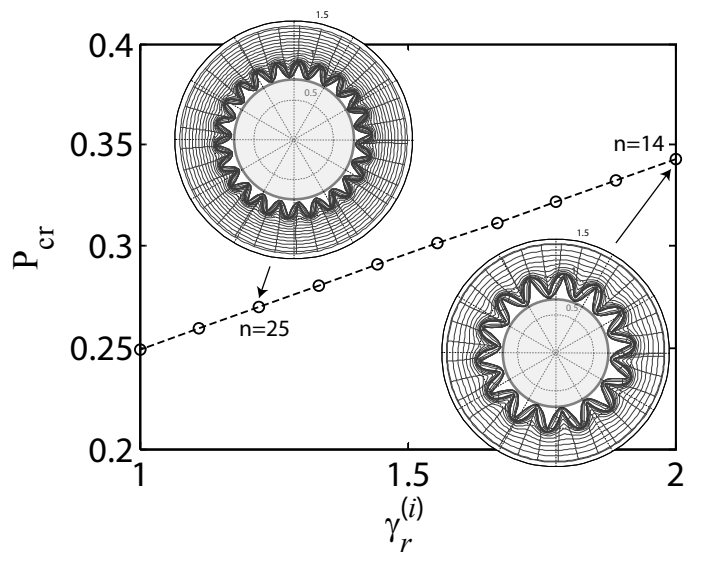

Figure 7: Critical pressure as a function of anisotropic growth of the inner layer. All other growth parameters are set to unity.

inner layer, creating a stabilizing tensile stress.) This seemingly counterintuitive result almost directly contradicts the findings of Wiggs et al. and hence highlights the important mechanical role of differential growth.

\subsection{Anisotropic growth}

In this section we consider anisotropic growth. For simplicity, we assume that the outer layer does not grow and explore buckling as a function of anisotropic growth in the inner layer by varying the ratio $\gamma_{r}^{(i)} / \gamma_{\theta}^{(i)}$. Thickening of the inner layer only occurs with radial growth. In Figure 7, the critical pressure is plotted for $\gamma_{\theta}^{(i)}=1$ fixed and $\gamma_{r}^{(i)}$ varying from 1 to 2 . As $\gamma_{r}^{(i)}$ increases, the inner layer becomes thicker - it doubles in size from $\gamma^{(i)}=1$ to 2 . Correspondingly, the buckling pressure increases, in a linear fashion. Again, there is a competition between mechanics and geometry: radial growth causes the inner layer to be relatively thicker - this is a stabilizing geometric effect but at the same time creates a radial compressive stress, which is destabilizing mechanically. In this case, since the inner layer was very thin to begin with, the geometric effect is stronger, and the resultant airway is more stable than before growth.

In terms of the degree of narrowing, with increasing growth the buckling mode decreases monotonically and significantly, from $n=27$ at $\gamma_{r}^{(i)}=1$ to $n=14$ at $\gamma_{r}^{(i)}=2$. Included in Figure 7 are the form of the deformation at the values $\gamma_{r}^{(i)}$ equals 1.2 and 2. Here the effect of buckling mode is apparent. In both cases, the inner radius at the point of bifurcation is about 0.87 , and both plots are produced using the same sized perturbation in the incremental deformation, but the airway narrowing is exaggerated at the point with the lower mode, $\gamma_{r}^{(i)}=2$. Defining the effective lumen as the area inside 
the innermost point of the folds (the shaded gray circles), we compute that this area is $18 \%$ greater in the case $\gamma_{r}^{(i)}=1.2$. Interestingly, if the anisotropic growth of the inner layer is accompanied by an isotropic swelling of the outer layer, the critical pressure is almost doubled, while the lumen area stays roughly the same (plot not shown).

\subsection{Fixed outer radius}

The model as we have presented it does not restrict outward growth. For instance in Case IV of Figure 6, notice that the outer edge is well beyond the location of the smooth muscle cells boundary before deformation (the outer radius of the reference state, equal to 1.5). This may not be realistic. It is likely that ASM, even before contracting, places a geometric constriction on the growth of the outer layer, so that the airway is restricted to grow radially outward, and must otherwise grow radially inward. The exact form of this constraint is complicated by the fact that the smooth muscle also grows during remodeling [23] and would require a detailed understanding of the growth relationships between the various layers of the airway. Nevertheless, some insight on the effect of a geometric constraint can be obtained if we fix the size of the outer radius during deformation. Mathematically, this changes the structure of the problem through the boundary condition since growth and pressure become inter-dependent. Fixing growth parameters automatically sets the pressure - a given growth creates pressure at the outer wall since the outer edge pushes against the smooth muscle. In this case, pressure is not a control parameter but is slaved to the growth parameters.

In Figure 8(a), we plot the bifurcation relationship between $\gamma^{(o)}$ and $\gamma^{(i)}$ for fixed outer radius. A given value of $\gamma^{(i)}$ and the corresponding $\gamma^{(o)}$ represent a form of growth which induces buckling. For each growth pair, the corresponding pressure is plotted in Figure 8(b).

There are different ways to view these plots and this version of the model. If we assume that the ASM interface is rigid and does not allow any outward growth, then Figure 8(a) shows the critical growth which induces buckling and Figure 8(b) is the pressure induced at the ASM interface due to the growth. In this sense growth itself is inducing buckling without any required contraction of the ASM. Alternatively, if the ASM layer is compliant, then some outward growth can occur, and when the ASM contracts the fixed outer radius requirement pushes the outer edge back to its original position. The biggest effect of the fixed outer radius condition is that the amount of growth is greatly restricted. Only the lower-left triangular region in Figure 8(a) constitutes valid growth. Viewing Figure 8(b), growth can be stabilizing or destabilizing. Buckling patterns are included at the select points, indicating again a trade off whereby the stronger airway is also narrower.

\subsection{Inverse problem}

It is clearly very difficult to determine experimentally the growth rates in the different layers. However, some geometric parameters, such as the thicknesses of normal airway and asthmatic airways are accessible. This raises the question: if we knew exactly how much thicker each layer of the airway wall became during remodeling and the pressure applied by the smooth muscle, could we determine the growth parameters 

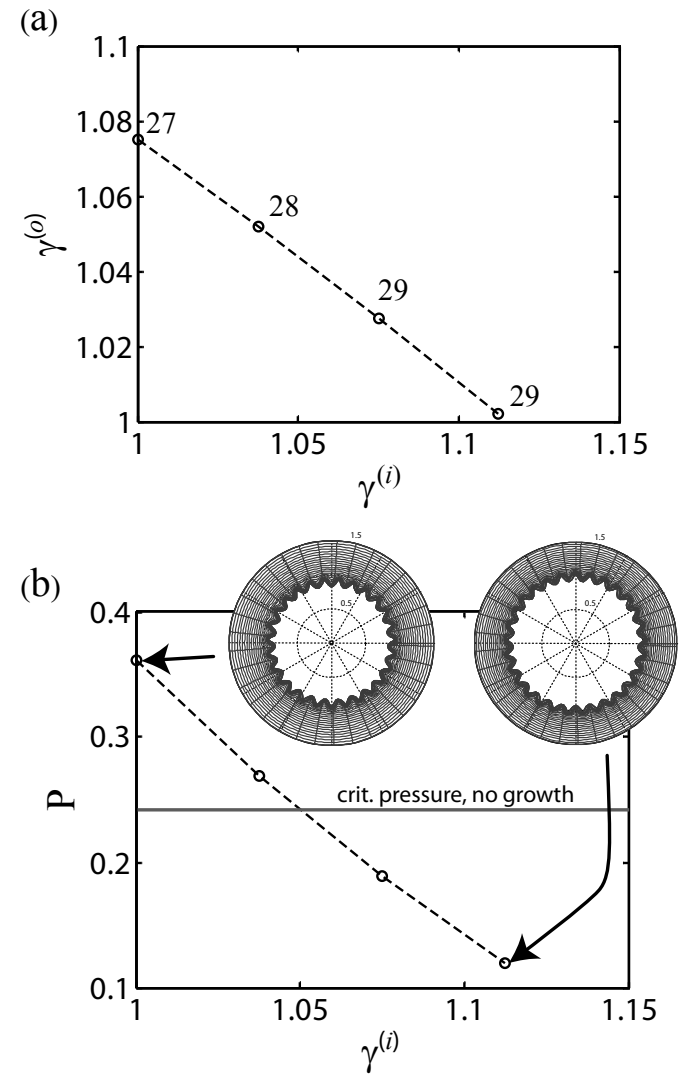

Figure 8: Critical outer growth $\gamma^{(o)}$ (a) or alternatively critical pressure (b) as a function of inner growth $\gamma^{(i)}$, for the model with fixed outer radius. The buckling mode at each point is marked in (a). Buckling patterns at the end points are provided in (b). 
and the amount of differential growth which occurred? We refer to this as the inverse problem: given the details of the deformation and the buckling, can we determine the type of growth which induced the deformation? Surprisingly, this can be done. A detailed analysis of this idea is given in a companion paper [35]. Mathematically, the argument can roughly be made by counting equations - there are 4 equations involved in determining the bifurcation of a bilayered cylinder. Since there are also 4 growth parameters, the inverse problem is well formulated, and a solution can in principle be found. A mathematical proof of this is lacking, but in all of the simulations we have attempted, a solution has been found.

We demonstrate here with a suggestive example. We start with a reference system in the absence of growth where the critical pressure is $P^{*}=0.2492$, the buckling mode is $n=27$, and the radii at the point of buckling are $a=0.8750, b=0.8974$, and $c=$ 1.4336. We now ask whether a growth could occur such that each layer is twice the thickness at the point of buckling and the buckling pressure is halved. We keep the same value of $b$ and double the relative thickness of each layer by taking $a=0.8526$ and $c=1.9698$. Then setting $P=P^{*} / 2=0.1246$, we find that the inverse problem has the solution $\gamma_{r}^{(i)}=1.92, \gamma_{\theta}^{(i)}=1.03, \gamma_{r}^{(o)}=2.35$, and $\gamma_{\theta}^{(o)}=1.05$. Notice that the ratio $\gamma_{r} / \gamma_{\theta}$ is greater than one in each layer. The interpretation is that if growth is faster in the radial direction in each layer at these particular values, the walls would grow such that the thickness at the point of buckling is doubled while at the same time buckling occurs at half the critical pressure as compared to the case with no growth. (Conversely, a solution could also be found with half the thickness and double the pressure.)

Again, the rationale for the existence of these solutions relates to the competing effects of residual stress and geometric effects. Given the large range of possibilities with anisotropic growth in two layers, there is sufficient flexibility in the growth variables that residual stress effects can be made dominant. For the example given, the growth causes a large compressive radial stress which dominates the geometric effect of the thicker inner layer. To further illustrate this effect, in Figure 9 we plot the radial stress profile in the tube. The dashed line is the stress due to growth alone, i.e. with no pressure applied, and the solid line is the stress at the point of bifurcation. We see that growth creates a strong compressive stress, so that only a small amount of additional pressure is required to induce buckling.

The point of this example is not to suggest that this exact value of growth may be occurring in airways, but rather to demonstrate the very significant impact that differential growth can have. If residual stress is generated, simply measuring airway dimensions and changes in dimensions could potentially be misleading as far as understanding mechanical stability.

\subsection{Airway size}

In order to focus on the effect of growth, we have thus far kept fixed the reference dimensions and stiffness ratio. Our choice of reference parameters was motivated by the general observation that the mucosa is significantly stiffer and thinner than the submucosa. However, one set of parameters certainly does not characterize all generations of the bronchial tree [22], and is also dependent on where you define the submucosa layer 


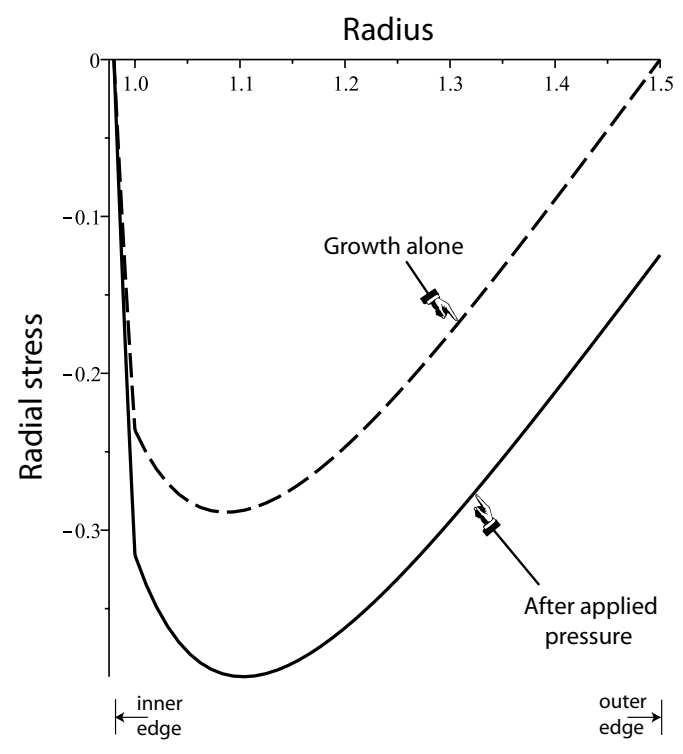

Figure 9: Radial stress as a function of position after unconstricted growth, i.e. with no applied pressure, (dashed line), and at the point of buckling. The compressive stress generated by the growth accounts for the decrease in stability even though the walls are thicker. 
as ending. In this section, we briefly explore the impact of varying those parameters.

To do this, we fix the growth parameters at $\gamma^{(i)}=1.4, \gamma^{(o)}=1.2$, and vary the inner layer thickness, outer layer thickness, and stiffness ratio. The result is plotted in Figure 10. As the inner layer thickness is increased (Figure 10(a)), the critical pressure increases and the buckling mode decreases, both quite significantly. This is expected, since the inner layer is stiffer and thinner. Note, however, the difference between this result and those of Section 3.1: here increasing the inner layer thickness in the reference dimensions is stabilizing, whereas increasing the inner layer thickness through differential growth can be destabilizing, as was shown in Section 3.1. Interestingly, an increase in thickness of the outer layer (Figure 10(b)) leads to a decrease in the critical pressure. The explanation for this is that, as explained in Section 3.1, the given growth parameters are destabilizing because the inner layer pushes against the outer layer and creates a destabilizing compressive stress. Increasing the outer layer thickness results in a harder body for the inner layer to push against, and thus leads to further destabilization. Nevertheless, we observe that the change in magnitude in critical pressure is quite small, and the buckling mode is essentially unaffected. This suggests that buckling behavior is not strongly dependent on small changes of submucosa thickness. Finally, as seen in Figure 10(c), varying the relative stiffness of the layers does not have a dramatic impact either, except in the limit when the stiffness ratio approaches unity.

\section{Discussion}

Similar to previous modeling attempts, in this paper we have studied the mechanical effect of airway wall thickness on mucosal folding and airway narrowing. The major addition in the present work which has not been included in any prior studies is that airway thickening occurs as a consequence of differential growth. Doing so, we have been able to study changes in material properties, in particular stability properties, due to the generation of residual stress.

Generally speaking, we have shown that differential growth can have a significant effect on airway buckling and therefore may be an important contributing factor in understanding the mechanical behavior of airways which have undergone airway remodeling. Note that only differential growth was considered, because it is the competition between geometry and mechanics that occurs as a result of the locally incompatible nature of differential growth that leads to interesting and counterintuitive behavior. Still, there are many ways in which differential growth could occur, and we have explored only a few possibilities here. Isotropic growth with differing rates in each layer led to the somewhat surprising conclusion that if the stiff mucosa grows at a faster rate than the soft submucosa, the airway actually becomes less stable. Anisotropic growth of the inner layer led to a strengthened airway but significantly decreased the buckling mode. Fixing the outer radius so as to account for the growth constriction of the smooth muscle greatly limited the amount of growth.

Our analysis provides a general framework to understand the mechanical effect of growth and remodeling, and our approach gives the ability to test hypotheses regarding growth and changes in airway stability. However, many open questions remain before anything definitive may be said. Differential growth provides a mechanism to ex- 


$$
\gamma^{(i)}=1.4, \gamma^{(o)}=1.2, \mu_{2}=1
$$

(a)

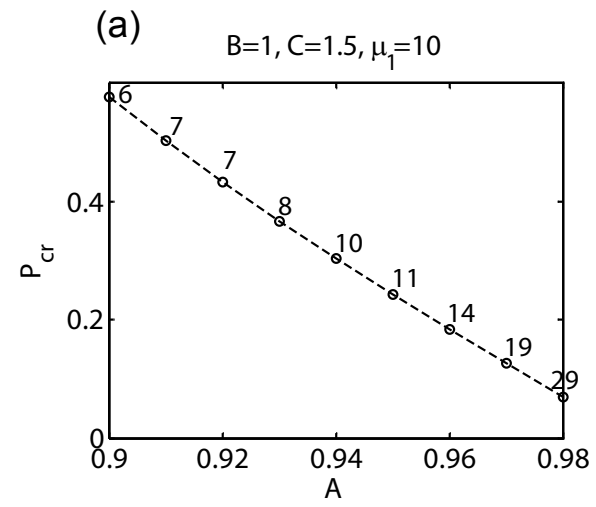

(b)

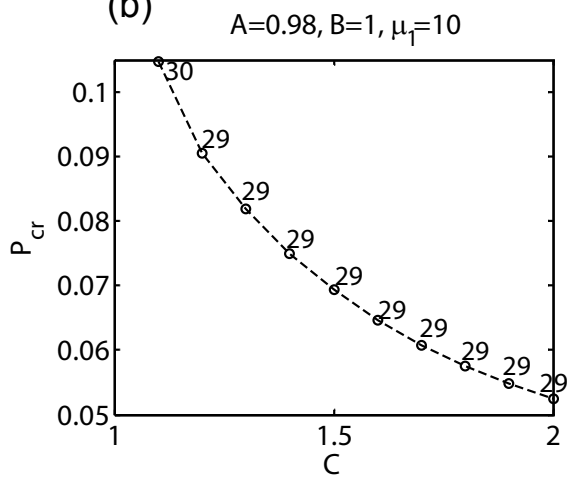

(c)

$A=0.98, B=1, C=1.5$

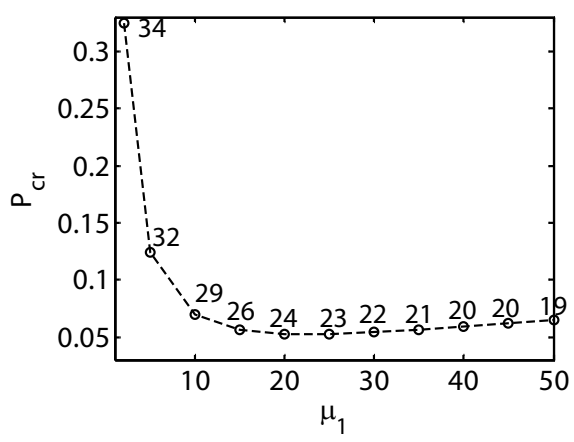

Figure 10: The buckling pressure for fixed growth parameters $\gamma^{(i)}=1.4, \gamma^{(o)}=1.2$, as the inner layer thickness (a), outer layer thickness (b), and stiffness (c), are varied. The buckling mode is labeled at each point. 
plain otherwise contradictory structural changes, for instance an airway wall becoming thicker and at the same time less stable. But airway remodeling involves more complex changes than just an increase of the mucosal and submucosal layers. The ASM layer also gains mass, and there is uncertainty as to whether the contractile potential changes [41]. Thus, whether the airways are actually less stable after remodeling is not yet established.

An obvious question is whether any direct evidence exists of differential growth in airway remodeling. We contend that more information is needed to conclude whether individual airway layers grow anisotropically, although anisotropic growth is known to occur in arteries [20,33]. On the other hand, it seems quite likely that different layers of the airway grow at different rates, since the material composition varies drastically, and there is good evidence to suggest that this form of differential growth is present in airway remodeling [44]. It should be noted, however, that in actuality growth is complex and cannot be fully captured by constant parameters $\gamma_{i}$ as utilized here, since the mass that is added may be of a different density or type than the normally present material [45].

If airway walls grow differentially and induce residual stress, as we have postulated here, this can be detected by opening-angle experiments, in which a ring of airway wall is cut radially. Any residual stress is relieved by the cut and the ring opens up. Such experiments are difficult, and have been carried out in only one study [31], where they reached the conclusion that human airways are essentially free of residual stress. However, it is important to note that none of the human lungs in that experimental study came from humans with asthma. This is a critical distinction, because our hypothesis is that normal airways are in a zero stress state, and that stress might be introduced through airway remodeling, in which case only asthmatic lungs would show an opening angle. Hence, opening angle experiments would need to be carried out on normal and asthmatic airways to confirm or deny the possibility of differential growth.

We now turn to the shortcomings of an idealized model. The airway is a complex structure, composed of multiple layers, which we have modeled as a bilayer cylindrical tube. This is certainly a simplification, although the purpose is to provide insight into the effect of growth in mucosal folding and an idealized model is sufficient to investigate these generic effects. The two dimensional nature of the model and the plane strain assumption are supported by the fact that folds in airways are observed as longitudinal ridges [56]. The linear stability analysis has the drawback that it only provides information on the deformation up to the point of the buckling. Nevertheless, knowledge of the buckling pressure is a good measure of the "strength" of an airway, and previous studies have shown that a tube becomes much more compliant after buckling [54], suggesting that narrowing occurs more rapidly after buckling. This is also apparent by an increased negative slope in pressure-area curves after buckling [54]. Thus, buckling pressure would seem to be a valid measure for investigating the mechanical impact of growth on airway narrowing. Moreover, the conclusion from prior models that buckling mode may be a significant indicator of airway narrowing seems to be supported by our analysis as well. Still, a proper analysis of occlusion and the change in cross sectional area would necessitate continuing the deformation beyond the point of buckling, which would require a much more computationally heavy numerical approach such as finite elements. Such an analysis coupled with growth would be an 
interesting direction for future modeling attempts.

The study we have presented is a preliminary examination of growth and mechanics in airways, and is largely qualitative. While we have attempted to use physiologically reasonable parameter values, exact values, in particular of the growth parameters, are unknown to us. Regarding the buckling pressure, which relates the normal force induced at the interface due to smooth muscle contraction, we can obtain a rough estimate of the magnitude given an estimation of the shear modulus. From a study on human airway walls [38], we estimate the shear modulus in the stiff mucosal layer, $\mu_{1}$, at around $120 \mathrm{kPA}$. Taking $P^{*} \approx 0.4$ as a typical value of buckling pressure for the dimensions considered here, this translates to an estimate of $1.6 \mathrm{kPA}$ as the amount of stress necessary to induce buckling. This is reasonable, as experiments have found that canine airways can generate pressures around $3 \mathrm{kPA}$ [14]. Note that this estimate is for a neo-Hookean material; the strain-stiffening Fung model leads to an increase in the critical stress.

Finally, the results presented here might also suggest a future direction of research. The structural changes associated with airway remodeling are generally considered uncontrollable and detrimental. However, if airways can be stimulated to grow in a particular way, growth can potentially both strengthen the airways against narrowing and reduce the degree of narrowing. In this sense, an analysis such as the one presented here could serve as a guideline for the type of growth necessary to achieve this.

Acknowledgments: This publication is based on work supported by Award No. KUKC1-013-04, made by King Abdullah University of Science and Technology (KAUST), and based in part upon work supported by the National Science Foundation under grant DMS-0907773 (AG). AG is a Wolfson/Royal Society Merit Award holder.

\section{Figure Legends}

Figure 1: Bilayer model of airway wall, consisting of a stiff and thin inner layer, the mucosa, and a soft and thick outer layer, the submucosa. Surrounding the submucosa is smooth muscle, which applies a normal pressure when it contracts.

Figure 2: Transformation of an area element under circumferential versus radial growth.

Figure 3: A comparison of pressure vs area for the symmetric deformation of a bilayered tube for 4 different forms of model. Parameters are $A=0.98, B=1, C=1.5$, and $\mu_{1} / \mu_{2}=10$.

Figure 4: Critical buckling pressure (a) and buckling mode (b) as a function of isotropic growth of the inner layer, for fixed isotropic growth in the outer layer, $\gamma^{(o)}=1.2$.

Figure 5: Critical buckling pressure (a) and buckling mode (b) as a function of isotropic growth of the outer layer, for fixed isotropic growth in the inner layer, $\gamma^{(i)}=1.2$.

Figure 6: Buckling patterns, or tube deformation, after bifurcation for the points marked I-IV in Figures 4(a) and 5(a). 
Figure 7: Critical pressure as a function of anisotropic growth of the inner layer. All other growth parameters are set to unity.

Figure 8: Critical outer growth $\gamma^{(o)}$ (a) or alternatively critical pressure (b) as a function of inner growth $\gamma^{(i)}$, for the model with fixed outer radius. The buckling mode at each point is marked in (a). Buckling patterns at the end points are provided in (b).

Figure 9: Radial stress as a function of position after unconstricted growth, i.e. with no applied pressure, (dashed line), and at the point of buckling. The compressive stress generated by the growth accounts for the decrease in stability even though the walls are thicker.

Figure 10: The buckling pressure for fixed growth parameters $\gamma^{(i)}=1.4, \gamma^{(o)}=1.2$, as the inner layer thickness (a), outer layer thickness (b), and stiffness (c), are varied. The buckling mode is labelled at each point.

\section{References}

[1] Bai A., D. H. Eidelman, J. C. Hogg, A. L. James, R. K. Lambert, M. S. Ludwig, J. Martin, D. M. McDonald, W. A. Mitzner, M. Okazawa, and al. et. Proposed nomenclature for quantifying subdivisions of the bronchial wall. J. Appl. Physiol., 77:1011-1014, 1994.

[2] Ben Amar M. and A. Goriely. Growth and instability in elastic tissues. J. Mech. Phys. Solids, 53:2284-2319, 2005.

[3] Bando K., D. Yamashita, and K. Ohba. Numerical Simulation for Mechanism of Airway Narrowing in Asthma. JSME International Journal Series C, 45(4):10201027, 2002.

[4] Boyce M. C. and E. M. Arruda. Constitutive models for rubber elasticity: a review. Rubber Chem. Technol., 73, 2000.

[5] Brook B. S., S. E. Peel, I. P. Hall, AZ Politi, J. Sneyd, Y. Bai, MJ Sanderson, and $\mathrm{OE}$ Jensen. A biomechanical model of agonist-initiated contraction in the asthmatic airway. Respiratory physiology \& neurobiology, 170(1):44-58, 2010.

[6] Donovan G.M. and M.H. Tawhai. A simplified model of airway narrowing due to bronchial mucosal folding. Respiratory physiology \& neurobiology, 171(2):144$150,2010$.

[7] Fung Y. C. What are the residual stresses doing in our blood vessels? Annals Biomed. Eng., 19:237-249, 1991.

[8] Fung Y. C. Biomechanics: Material Properties of Living Tissues. Springer, New York, 1993. 
[9] Gleason R. L. and J. D. Humphrey. A mixture model of arterial growth and remodeling in hypertension: altered muscle tone and tissue turnover. J. Vasc. Research, 41:352-363, 2004.

[10] Goriely A., M. Destrade, and M. Ben Amar. Instabilities in elastomers and in soft tissues. The Quarterly Journal of Mechanics and Applied Mathematics, 59(4):615, 2006.

[11] Goriely A., and D. E. Moulton. Morphoelasticity - a theory of elastic growth. In M. Mueller M. Ben Amar, A. Goriely, editor, New Trends in the Physics and Mechanics of Biological Systems. Oxford University Press, 2010.

[12] Goriely A., D. E. Moulton, and R. Vandiver. Elastic cavitation, tube hollowing, and differential growth in plants and biological tissues. EPL (Europhysics Letters), 91:18001, 2010.

[13] Goriely A. and R. Vandiver. On the mechanical stability of growing arteries. IMA J. Appl. Math., Accepted:1-22, 2010.

[14] Gunst S. J. and J. Q. Stropp. Pressure-volume and length-stress relationships in canine bronchi in vitro. Journal of Applied Physiology, 64(6):2522, 1988.

[15] Hill M.J.,T.A. Wilson, and R.K. Lambert. Effects of surface tension and intraluminal fluid on mechanics of small airways. Journal of Applied Physiology, 82(1):233, 1997.

[16] Holzapfel G. A. , T. C. Gasser, and R. W. Ogden. Comparison of a multi-layer structural model for arterial walls with a Fung-type model, and issues of material stability. J. Biomech. Engineering, 126:264, 2004.

[17] Holzapfel G. A. and R.W. Ogden. Modelling the layer-specific three-dimensional residual stresses in arteries, with an application to the human aorta. Journal of the Royal Society Interface, 7(787-799), 2010.

[18] Hrousis C. A., B. R. Wiggs, J. M. Drazen, D. M. Parks, and R. D. Kamm. Mucosal folding in biologic vessels. J. Biomech. Eng., 124(4):334-341, 2002.

[19] Huber H. L. and K. K. Koessler. The pathology of bronchial asthma. Arch. Intern. Med., 30, 1922.

[20] Humphrey J. D. . Continuum biomechanics of soft biological tissues. Proc. Roy. Soc. Lond. A, 459:3-46, 2003.

[21] James A. Airway remodeling in asthma. Curr. Opin. Pulm. Med., 11:1-6, 2004.

[22] James A. L., P. D. Paré, and J. C. Hogg. The mechanics of airway narrowing in asthma. Am. Rev. Respir. Dis., 139(1):242-246, 1989.

[23] King G. G., P. D. Paré, and C. Y. Seow. The mechanics of exaggerated airway narrowing in asthma: the role of smooth muscle. Resp. Physiol., 118:1-13, 1999. 
[24] Kuwano K., C. H. Bosken, P. D. Paré, T. R. Bai, B. R. Wiggs, and J. C. Hogg. Small airways dimensions in asthma and in chronic obstructive pulmonary disease. Am. Rev. Respir. Dis., 148(5):1220-1225, 1993.

[25] Lambert R. K. Role of bronchial basement membrane in airway collapse. J. Appl. Physiol., 71:666-673, 1991.

[26] Lambert R. K., S. L. Codd, M. R. Alley, and R. J. Pack. Physical determinants of bronchial mucosal folding. J. Appl. Physiol., 77:1206-1216, 1994.

[27] Lee M. M. L. and S. Chien. Morphologic effects of pressure changes on canine carotid artery endothelium as observed by scanning electron microscopy. The Anatom. Rec., 194:1-14, 1978.

[28] Liao D., J. Zhao, J. Yang, and H. Gregersen. The oesophageal zero-stress state and mucosal folding from a giome perspective. World J. Gastroenterol, 13(9):13471351, 2007.

[29] Lin L. E. and L. Taber. A model for stress-induced growth in the developing heart. J. Biomech. Engineering, 117:343-349, 1995.

[30] Lu X., J. Zhao, and H. Gregersen. Small intestinal morphometric and biomechanical changes during physiological growth in rats. J. Biomech., 38, 2005.

[31] McKay K. O., B. R. Wiggs, P. D. Paré, and R. D. Kamm. Zero-stress state of intra- and extraparenchymal airways from human, pig, rabbit, and sheep lung. $J$. Appl. Physiol., 92:1261-1266, 2002.

[32] McParland B. E., P. T. Macklem, and P. D. Pare. Airway hyperresponsiveness: From molecules to bedside. J. Appl. Physiol., 95:426-434, 2003.

[33] Menzel A. Modelling of anisotropic growth in biological tissues. Biomechanics and modeling in mechanobiology, 3(3):147-171, 2005.

[34] Moore B. J., C. C. Hilliam, L. M. Verburgt, B. R. Wiggs, S. Vedal, and P. D. Paré. Shape and position of the complete dose-response curve for inhaled methacholine in normal subjects. Am. J. Respir. Crit. Care. Med., 154, 1996.

[35] Moulton D. E. and A. Goriely. Circumferential buckling instability of a growing cylindrical tube. J. Mech. Phys. Solids. preprint.

[36] Niimi A., H. Matsumoto, R. Amitani, Y. Nakano, M. Mishima, M. Minakuchi, K. Nishimura, H. Itoh, and T. Izumi. Airway wall thickness in asthma assessed by computed tomography. Am. J. Respir. Crit. Care Med., 162:1518-1523, 2000.

[37] Noble P. B., A. Sharma, P. K. McFawn, and H. W. Mitchell. Airway narrowing in porcine bronchi with and without lung parenchyma. European Respiratory Journal, 26(5):804, 2005.

[38] Ogawa Y. Study on the strength of human lower air-passages. J. Kyoto Prefect Univ. Med, 66:781-800, 1959. 
[39] Ogden R. W. Non-linear elastic deformation. Dover, New york, 1984.

[40] Ogden R. W. and C. A. J. Schulze-Bauer. Phenomenological and structural aspects of the mechanical response of arteries. ASME Applied Mech., 242:125-140, 2000.

[41] Paré P. D., B. E. McParland, and C. Y. Seow. Structural basis for exaggerated airway narrowing. Can. J. Physiol. Pharmacol., 85(7):635-638, 2007.

[42] Politi A. Z., G. M. Donovan, M. H. Tawhai, M. J. Sanderson, A. M. Lauzon, J. H. T. Bates, and J. Sneyd. A multiscale, spatially distributed model of asthmatic airway hyper-responsiveness. Journal of Theoretical Biology, 2010.

[43] Redington A. E. and P. H. Howarth. Airway wall remodelling in asthma. Thorax, 52:310-312, 1997.

[44] Roberts C. R. Is asthma a fibrotic disease? Chest, 107:111S-117S, 1995.

[45] Roche W.R., J. H. Williams, R. Beasley, and S. T. Holgate. Subepithelial fibrosis in the bronchi of asthmatics. The Lancet, 333(8637):520-524, 1989.

[46] Rodriguez E. K., A. Hoger, and A. McCulloch. Stress-dependent finite growth in soft elastic tissues. J. Biomech, 27:455-467, 1994.

[47] Seow C. Y., L. Wang, and P. D. Pare. Airway narrowing and internal structural constraints. Journal of Applied Physiology, 88(2):527, 2000.

[48] Taber L. A. Biomechanics of growth, remodeling and morphogenesis. Appl. Mech. Rev., 48:487-545, 1995.

[49] Taber L. A. Biomechanical growth laws for muscle tissues. J. Theor. Biol., 193:201-213, 1998.

[50] Vandiver R. and A. Goriely. Tissue tension and axial growth of cylindrical structures in plants and elastic tissues. Europhys. Lett., 84:58004, 2008.

[51] Vignola A. M., F. Mirabella, G. Costanzo, R. Di Giorgi, M. Gjomarkaj, V. Bellia, and G. Bonsignore. Airway remodeling in asthma. Chest, 123(3):417S-422S, 2003.

[52] Wang A. S. D. and A. Ertepinar. Stability and vibrations of elastic thick-walled cylindrical and spherical shells subjected to pressure. Int. J Non-Linear Mech., 7:539-555, 1972.

[53] Wang I., A. Z. Politi, N. Tania, Y. Bai, M. J. Sanderson, and J. Sneyd. A mathematical model of airway and pulmonary arteriole smooth muscle. Biophysical journal, 94(6):2053-2064, 2008.

[54] Wiggs B. R., C. A. Hrousis, J. M. Drazen, and R. D. Kamm. On the mechanism of mucosal folding in normal and asthmatic airways. J. Appl. Physiol, 83:1814$1821,1997$. 
[55] Xu G., P. V. Bayly, and L. A. Taber. Residual stress in the adult mouse brain. Biomechanics and modeling in mechanobiology, 8(4):253-262, 2009.

[56] Yager D., T. Cloutier, H. Feldman, J. Bastacky, J. M. Drazen, and R. D. Kamm. Airway surface liquid thickness as a function of lung volume in small airways of the guinea pig. J. Appl. Physiol., 77(5):2333-2340, 1994.

[57] Yang W., T. C. Fung, K. S. Chian, and C. K. Chong. Instability of the twolayered thick-walled esophageal model under the external pressure and circular outer boundary condition. J. Biomech., 40, 2007. 



\section{RECENT REPORTS}

25/10 Preconditioning for Allen-Cahn Variational Inequalities with Non- Blank Local Constraints

Sarbu

Stoll

26/10 On an evolution equation for sand dunes

Ellis

Fowler

27/10 On Liquid Films on an Inclined Plate

Benilov

Chapman

McLoed

Ockendon

Zubkov

28/10 An a posteriori error analysis of a mixed finite element Galerkin approximation to second order linear parabolic problems

Memon

Nataraj

Pani

29/10 A Priori Error Estimates for Semidiscrete Finite Element Approx-

Goswami imations to Equations of Motion Arising in Oldroyd Fluids of Order One

30/10 The Landau-de Gennes theory of nematic liquid crystals: Uniaxiality versus Biaxiality

31/10 The Radial-Hedgehog Solution in Landau-de Gennes' theory

Majumdar

32/10 Nonlinear instability in flagellar dynamics: a novel modulation mechanism in sperm migration?

Gadelha

Gaffney

Smith

Kirkman-Brown

33/10 Error bounds on block GaussSeidel solutions of coupled multiphysics problem

Whiteley

Gillow

Tavener

Walter

34/10 A random projection method for sharp phase boundaries in lattice Reis Boltzmann simulations

Dellar

35/10 Regularized Particle Filter with Langevin Resampling Step

Duan

Farmer

Moroz

36/10 Sequential Inverse Problems Bayesian Principles and the Logistic Map Example

Duan

Farmer

Moroz

37/10 Circumferential buckling instability of a growing cylindrical tube

Moulton

Goriely

38/10 Preconditioners for state constrained optimal control problems

Stoll with Moreau-Yosida penalty function

Wathen

39/10 Local synaptic signaling enhances the stochastic transport of

Newby motor-driven cargo in neurons

Bressloff 
40/10 Convection and Heat Transfer in Layered Sloping Warm-Water Aquifer

McKibbin

Hale

Style

Walters

41/10 Optimal Error Estimates of a Mixed Finite Element Method for

Goswami

Parabolic Integro-Differential Equations with Non Smooth Initial

Pani

Data

Yadav

42/10 On the Linear Stability of the Fifth-Order WENO Discretization

Motamed

Macdonald

Ruuth

43/10 Four Bugs on a Rectangle

Chapman

Lottes

Trefethen

44/10 Mud peeling and horizontal crack formation in drying clay

Style

Peppin

Cocks

45/10 Binocular Rivalry in a Competitive Neural Network with Synaptic

Kilpatrick Depression

Bressloff

46/10 A theory for the alignment of cortical feature maps during devel-

Bressloff opment

Oster

47/10 All-at-Once Solution if Time-Dependent PDE-Constrained Opti-

Stoll misation Problmes

Wathen

Copies of these, and any other OCCAM reports can be obtained from:

Oxford Centre for Collaborative Applied Mathematics

Mathematical Institute

24 - 29 St Giles'

Oxford

OX1 3LB

England

www.maths.ox.ac.uk/occam 